\title{
Thermal simulation of heterogeneous structural components in microelectronic devices
}

\author{
V.I. Gavrysh, D.V. Fedasyuk \\ Lviv Polytechnic National University \\ E-mail: fedasyuk@lp.edu.ua
}

\begin{abstract}
The steady state nonlinear problem of thermal conduction for isotropic strip with foreign rectangular inclusion that heats as an internal thermal source with heat dissipation has been considered. The methodology to solve this problem and its application for the specific dependence of the thermal-conductivity coefficients on temperature has been offered.
\end{abstract}

Keywords: isotropic, thermal conduction, foreign inclusion, thermosensitive, heat dissipation.

Manuscript received 03.06.10; accepted for publication 02.12.10; published online 30.12.10.

\section{Introduction}

In the design process of individual junctions and structural components for microelectronic devices, there appears the necessity to mathematically model thermal processes in thermosensitive structures with foreign inclusions that are one of the important parts in modern engineering researches. The construction and study of mathematical models for thermoconductivity processes in thermosensitive structures with foreign inclusions requires the development of new efficient methods to solve boundary mathematical physics problems of both theoretical and practical significance.

The thermal process is nonlinear, and the presence of foreign inclusions complicates these mathematical models but increases the research accuracy, which is topical as to their heat resistance. This requires new algorithms for construction and appropriate software development for the temperature condition analysis of individual junctions and structural elements of electronic devices.

In the process of solving the boundary thermal conductivity problems, it is important to consider the dependence of thermal-and-physical characteristics on temperature, which allows describing the distribution of temperature fields and their gradients in the systems under consideration to be more accurate and precise.

The approximate analytical solution for half-space with a small foreign heat dissipating cylindrical inclusion was obtained in the paper [1]. The analytical solution for the isotropic strip with the rectangular inclusion of an arbitrary size is produced in the paper [2]. General thermal conductivity equations for the thermosensitive piecewise homogeneous solids are presented in the works $[3,4]$.

\section{Problem statement}

Due to the complex structure of separate junctions and construction elements in modern electronic devices the necessity in modeling of thermosensitive systems with foreign inclusions has arisen. Therefore we consider the isotropic thermosensitive strip with rectangular inclusion with square $4 h l$ referred to the Cartesian coordinate system $O x y$ with its origin in the center of inclusion (Figure). At the coupling intervals $L_{x}^{ \pm}=\{( \pm h, y):|y| \leq l\}, \quad L_{y}^{ \pm}=\{(x, \pm l):|x| \leq h\}, \quad$ the conditions of ideal thermal contact are fulfilled. At the strip edges $K_{+}=\left\{\left(x, l+d_{1}\right):|x|<\infty\right\}$, $K_{-}=\{(x, \pm l):|x| \leq h\}$, the conditions of convective heat exchange with the constant environment temperature $t_{c}$ are given. In the inclusion area $\Omega_{0}=\{(x, y):|x| \leq h,|y| \leq l\}$ with uniformly distributed internal heat sources, the power is $q_{0}$. 


\section{Partially linearized initial boundary problem}

The distribution of steady state temperature field $t(x, y)$ in the thermosensitive strip is obtained by solving the nonlinear thermal conductivity equation $[3,4]$

$\frac{\partial}{\partial x}\left[\lambda(x, y, t) \cdot \frac{\partial t}{\partial x}\right]+\frac{\partial}{\partial y}\left[\lambda(x, y, t) \cdot \frac{\partial t}{\partial y}\right]=$

$=-q_{0} \cdot N(x, h) \cdot N(y, l)$,

taking into account the following boundary conditions:

$\left.\lambda_{1}(t) \cdot \frac{\partial t}{\partial y}\right|_{y=l+d_{1}}=-\alpha_{+} \cdot\left(\left.t\right|_{y=l+d_{1}}-t_{c}\right)$,

$\left.\lambda_{1}(t) \cdot \frac{\partial t}{\partial y}\right|_{y=-l-d_{2}}=\alpha_{-} \cdot\left(\left.t\right|_{y=-l-d_{2}}-t_{c}\right)$,

$\left.t\right|_{|x| \rightarrow \infty}=0,\left.\frac{\partial t}{\partial x}\right|_{|x| \rightarrow \infty}=0$,

where $\lambda(x, y, t)=\lambda_{1}(t)+\left[\lambda_{0}(t)-\lambda_{1}(t)\right] \cdot N(x, h) \cdot N(y, l)$ is the thermal conductivity coefficient of the heterogeneous strip; $\lambda_{0}, \lambda_{1}-$ heat conduction coefficients of the inclusion and strip; $\alpha_{ \pm}-$coefficient of heat elimination from the strip edges $K_{ \pm}$; $N(\zeta, \eta)=S_{-}(\zeta+\eta)-S_{+}(\zeta-\eta) ; S_{ \pm}(\zeta)-$ asymmetric unit functions.

Input the function [5]

$$
\begin{aligned}
& \vartheta=\int_{0}^{t(x, y)} \lambda_{1}(\zeta) d \zeta+\left\{\int_{t( \pm h, y)}^{t(x, y)}\left[\lambda_{0}(\zeta)-\lambda_{1}(\zeta)\right] d \zeta \cdot N(y, l)-\right. \\
& -\int_{t( \pm h,-l)}^{t(x,-l)}\left[\lambda_{0}(\zeta)-\lambda_{1}(\zeta)\right] d \zeta \cdot S_{-}(y+l)+ \\
& \left.+\int_{t( \pm h, l)}^{t(x, l)}\left[\lambda_{0}(\zeta)-\lambda_{1}(\zeta)\right] d \zeta \cdot S_{+}(y-l)\right\} \cdot S_{-}(h-|x|)
\end{aligned}
$$

and obtain by differentiating with respect to $x$ and $y$ :

$\frac{\partial \vartheta}{\partial x}=\lambda(t, x, y) \frac{\partial t}{\partial x}+$

$+\left\{\left.\left[\left(\lambda_{0}(t)-\lambda_{1}(t)\right) \frac{\partial t}{\partial x}\right]\right|_{y=l} \cdot S_{+}(y-l)-\right.$

$\left.-\left[\left(\lambda_{0}(t)-\lambda_{1}(t)\right) \frac{\partial t}{\partial x}\right]||_{y=-l} \cdot S_{-}(y+l)\right\} \cdot S_{-}(h-|x|)$,

$\frac{\partial \vartheta}{\partial y}=\lambda(t, x, y) \frac{\partial t}{\partial x}-\left.\left\{\left(\lambda_{0}(t)-\lambda_{1}(t)\right) \frac{\partial t}{\partial y}\right\}\right|_{|x|=h} \times$

$\times S_{-}(h-|x|) \cdot N(y, l)$.

Considering the expressions (4) and equation (1), we can write:

$$
\begin{aligned}
& \Delta \vartheta=\frac{\partial}{\partial x}\left\{\left[\left.\left(\left(\lambda_{0}(t)-\lambda_{1}(t)\right) \cdot \frac{\partial t}{\partial x}\right)\right|_{y=l} \cdot S_{+}(y-l)-\right.\right. \\
& \left.\left.-\left.\cdot\left(\left(\lambda_{0}(t)-\lambda_{1}(t)\right) \cdot \frac{\partial t}{\partial x}\right)\right|_{y=-l} \cdot S_{-}(y+l)\right] \cdot S_{-}(h-|x|)\right\}- \\
& -q_{0} \cdot S_{-}(h-|x|) \cdot N(y, l)- \\
& -\frac{\partial}{\partial y}\left\{\left[\left(\lambda_{0}(t)-\lambda_{1}(t)\right) \cdot \frac{\partial t}{\partial y}\right]_{|x|=h} \cdot S_{-}(h-|x|) \cdot N(y, l)\right\} .
\end{aligned}
$$

Here, $\Delta$ is the Laplace operator.

$$
\begin{aligned}
& \left.\frac{\partial \vartheta}{\partial y}\right|_{y=l+d_{1}}=-\alpha_{+} \cdot\left(\left.t\right|_{y=l+d_{1}}-t_{c}\right),\left.\quad \vartheta\right|_{|x| \rightarrow \infty}=0, \\
& \left.\frac{\partial \vartheta}{\partial x}\right|_{|x| \rightarrow \infty}=0,\left.\quad \frac{\partial \vartheta}{\partial y}\right|_{y=-l-d_{2}}=-\alpha_{-}\left(\left.t\right|_{y=-l-d_{2}}-t_{c}\right) .
\end{aligned}
$$

Hence, the nonlinear boundary equations (1), (2) with application of the input function (3) can be reduced to a partially linearized problem (5), (6).

\section{Fully linearized boundary problem}

Let us approximate $t( \pm h, y), t(x, \pm l), t\left(x, l+d_{1}\right)$, $t\left(x,-l-d_{2}\right)$ functions with the following expressions

$$
\begin{aligned}
& t( \pm h, y)=t_{1}^{( \pm h)}+\sum_{j=1}^{n-1}\left(t_{j+1}^{( \pm h)}-t_{j}^{( \pm h)}\right) \cdot S_{-}\left(y-y_{j}\right), \\
& t(x, \pm l)=t_{1}^{( \pm l)}+\sum_{j=1}^{n-1}\left(t_{j+1}^{( \pm l)}-t_{j}^{( \pm l)}\right) \cdot S_{-}\left(x-x_{j}\right), \\
& t\left(x, l+d_{1}\right)=t_{1}^{(+)}+\sum_{k=1}^{m-1}\left(t_{k+1}^{(+)}-t_{k}^{(+)}\right) S_{-}\left(x-x_{k}\right), \\
& t\left(x,-l-d_{2}\right)=t_{1}^{(-)}+\sum_{k=1}^{m-1}\left(t_{k+1}^{(-)}-t_{k}^{(-)}\right) S_{-}\left(x-x_{k}\right),
\end{aligned}
$$

where $t_{j}^{( \pm h)}, t_{j}^{( \pm l)}, t_{k}^{(+)}, t_{k}^{(-)}$are unknown approximating temperature values $\left.y_{j} \in\right]-l, l\left[; \quad y_{1}<y_{2}<\ldots<y_{n-1}\right.$; $\left.x_{j} \in\right]-h, 0[] 0,, h\left[; \quad x_{1}<x_{2}<\ldots<x_{n-1} ; \quad x_{k} \in\right] 0, x_{*}[$; $x_{1}<x_{2}<\ldots<x_{m-1} ; \quad x_{*}$ is the abscissa value for which the temperature is practically equal to $t_{c}$ (is obtained from the relevant linear problem); $n$ - number of partitions in the intervals $]-l, l[]-h,, 0[$ and $] 0, h[, m-$ number of partitions in the interval $] 0, x_{*}[$.

Substituting expressions (7) in the equation (5) and boundary conditions (6) at the edges of strip $K_{ \pm}$, a linear boundary problem for finding the function $\vartheta$ is obtained 
$\Delta \vartheta=\sum_{j=1}^{n-1}\left(t_{j+1}^{(l)}-t_{j}^{(l)}\right) \cdot\left[\lambda_{0}\left(t_{j+1}^{(l)}\right)-\lambda_{1}\left(t_{j+1}^{(l)}\right)\right] \cdot \delta_{-}^{\prime}\left(x-x_{j}\right) \cdot S_{+}(y-l)-$ $-\sum_{j=1}^{n-1}\left(t_{j+1}^{(-l)}-t_{j}^{(-l)}\right) \cdot\left[\lambda_{0}\left(t_{j+1}^{(-l)}\right)-\lambda_{1}\left(t_{j+1}^{(-l)}\right)\right] \cdot \delta_{-}^{\prime}\left(x-x_{j}\right) S_{-}(y+l)-$ $-\sum_{j=1}^{n-1}\left(t_{j+1}^{( \pm h)}-t_{j}^{( \pm h)}\right) \cdot\left[\lambda_{0}\left(t_{j+1}^{( \pm h)}\right)-\lambda_{1}\left(t_{j+1}^{( \pm h)}\right)\right] \cdot S_{-}(h-|x|) \cdot \delta_{-}^{\prime}\left(y-y_{j}\right)-$ $-q_{0} \cdot S_{-}(h-|x|) \cdot N(y, l)$,

$\left.\frac{\partial \vartheta}{\partial y}\right|_{y=l+d_{1}}=-\alpha_{+} \cdot\left[t_{1}^{(+)}+\sum_{k=1}^{m-1}\left(t_{k+1}^{(+)}-t_{k}^{(+)}\right) \cdot S_{-}\left(x-x_{k}\right)-t_{c}\right]$,

$\left.\frac{\partial \vartheta}{\partial y}\right|_{y=-l-d_{2}}=\alpha_{-} \cdot\left[t_{1}^{(-)}+\sum_{k=1}^{m-1}\left(t_{k+1}^{(-)}-t_{k}^{(-)}\right) \cdot S_{-}\left(x-x_{k}\right)-t_{c}\right]$,

$\left.\vartheta\right|_{|x| \rightarrow \infty}=0,\left.\frac{\partial \vartheta}{\partial x}\right|_{|x| \rightarrow \infty}=0$,

where $\delta_{-}(\zeta)$ is the asymmetric Dirac delta function .

\section{Construction of analytical solution to the linear boundary problem (8), (9)}

Applying the Fourier integral transform by the $x$ coordinate for the boundary problem (8), (9), we obtain the ordinary differential equations with constant coefficients:

$$
\begin{aligned}
& \frac{\partial^{2} \vartheta}{\partial y^{2}}-\xi^{2} \bar{\vartheta}=i \sqrt{\frac{1}{2 \pi}} \cdot\left\{\xi \cdot \left[\sum_{j=1}^{n-1} e^{i \xi x_{j} \cdot\left(\lambda_{0}\left(t_{j+1}^{(-l)}\right)-\lambda_{1}\left(t_{j+1}^{(-l)}\right)\right) \times}\right.\right. \\
& \times\left(t_{j+1}^{(-l)}-t_{j}^{(-l)}\right) \cdot S_{-}(y+l)- \\
& \left.-\sum_{j=1}^{n-1} e^{i \xi x} \cdot\left(\lambda_{0}\left(t_{j+1}^{(+l)}\right)-\lambda_{1}\left(t_{j+1}^{(+l)}\right)\right) \cdot\left(t_{j+1}^{(+l)}-t_{j}^{(+l)}\right) \cdot S_{+}(y-l)\right]+ \\
& +\frac{2 \operatorname{sh} i \xi h}{\xi} \cdot\left[q_{0} \cdot N(y, l)+\sum_{j=1}^{n-1}\left(\lambda_{0}\left(t_{j+1}^{( \pm h)}\right)-\lambda_{1}\left(t_{j+1}^{( \pm h)}\right)\right) \times\right. \\
& \left.\left.\times\left(t_{j+1}^{( \pm h)}-t_{j}^{( \pm h)}\right) \cdot \delta_{-}^{\prime}\left(y-y_{j}\right)\right]\right\}
\end{aligned}
$$

and the following boundary conditions:

$$
\begin{aligned}
& \left.\frac{\partial \bar{\vartheta}}{\partial y}\right|_{y=l+d_{1}}=-\frac{i \alpha_{+}}{\sqrt{2 \pi \xi}} \cdot \sum_{k=1}^{m-1}\left(t_{k+1}^{(+)}+t_{k}^{(+)}\right) \cdot e^{i \xi x_{k}}, \\
& \left.\frac{\partial \bar{\vartheta}}{\partial y}\right|_{y=-l-d_{2}}=-\frac{i \alpha_{-}}{\sqrt{2 \pi \xi}} \cdot \sum_{k=1}^{m-1}\left(t_{k+1}^{(-)}+t_{k}^{(-)}\right) \cdot e^{i \xi x_{k}},
\end{aligned}
$$

where $\bar{\vartheta}=\sqrt{\frac{1}{2 \pi}} \cdot \int_{-\infty}^{\infty} e^{i \xi \xi x} \vartheta d x-$ function $\vartheta$ transformant; $i=\sqrt{-1}$.
The general solution to the equation (10) is:

$\bar{\vartheta}=C_{1} \cdot e^{\xi y}+C_{2} \cdot e^{-\xi y}-\sqrt{\frac{1}{2 \pi}} \cdot \frac{i}{\xi} \times$

$\times\left\{\sum_{j=1}^{n-1} e^{i \xi x_{j}} \cdot\left[\lambda_{0}\left(t_{j+1}^{(-l)}\right)-\lambda_{1}\left(t_{j+1}^{(-l)}\right)\right] \times\right.$

$\times\left(t_{j+1}^{(-l)}-t_{j}^{(-l)}\right) \cdot(1-\operatorname{ch} \xi(y+l)) \cdot S_{-}(y+l)-$

$-\sum_{j=1}^{n-1} e^{i \xi x_{j}} \cdot\left[\lambda_{0}\left(t_{j+1}^{(l)}\right)-\lambda_{1}\left(t_{j+1}^{(l)}\right)\right] \times$

$\times\left(t_{j+1}^{(l)}-t_{j}^{(l)}\right) \cdot(1-\operatorname{ch} \xi(y-l)) \cdot S_{+}(y-l)-$

$-2 \operatorname{sh} i \xi h \cdot\left[\sum_{j=1}^{n-1}\left[\lambda_{0}\left(t_{j+1}^{( \pm h)}\right)-\lambda_{1}\left(t_{j+1}^{( \pm h)}\right)\right] \times\right.$

$\times\left(t_{j+1}^{( \pm h)}-t_{j}^{( \pm h)}\right) \operatorname{ch} \xi\left(y-y_{j}\right) \cdot S_{-}\left(y-y_{j}\right)--\frac{q_{0}}{\xi^{2}} \times$

$\left.\left.\times\left(N(y, l)-\operatorname{ch} \xi(y+l) \cdot S_{-}(y+l)+\operatorname{ch} \xi(y-l) \cdot S_{+}(y-l)\right)\right]\right\}$.

Here $C_{1}$ and $C_{2}$ are integrating constants.

Having applied the boundary conditions (11), a partial solution to the problem (10), (11) is obtained

$\bar{\vartheta}=-\sqrt{\frac{1}{2 \pi}} \cdot \frac{i}{\xi} \cdot\left\{\sum_{j=1}^{n-1} e^{i \xi x_{j}} \cdot\left[\lambda_{0}\left(t_{j+1}^{(-l)}\right)-\lambda_{1}\left(t_{j+1}^{(-l)}\right)\right] \cdot\left(t_{j+1}^{(-l)}-t_{j}^{(-l)}\right) \times\right.$

$\times\left[(1-\operatorname{ch} \xi(y+l)) S_{-}(y+l)+\frac{\operatorname{sh} \xi\left(2 l+d_{1}\right)}{\operatorname{sh} \xi\left(2 l+d_{1}+d_{2}\right)} \operatorname{ch} \xi\left(y+l+d_{2}\right)\right]-$

$-\sum_{j=1}^{n-1} e^{i \xi x_{j}} \cdot\left[\lambda_{0}\left(t_{j+1}^{(l)}\right)-\lambda_{1}\left(t_{j+1}^{(l)}\right)\right]\left(t_{j+1}^{(l)}-t_{j}^{(l)}\right) \times$

$\times\left[(1-\operatorname{ch} \xi(y-l)) S_{+}(y-l)+\frac{\operatorname{sh} \xi d_{2}}{\operatorname{sh} \xi\left(2 l+d_{1}+d_{2}\right)} \operatorname{ch} \xi\left(y+l+d_{2}\right)\right]-$

$-2 \operatorname{sh} i \xi h \cdot\left[\sum_{j=1}^{n-1}\left[\left(t_{j+1}^{( \pm h)}-t_{j}^{( \pm h)}\right) \cdot\left(\lambda_{0}\left(t_{j+1}^{( \pm h)}\right)-\lambda_{1}\left(t_{j+1}^{( \pm h)}\right)\right) \times\right.\right.$

$\times\left(\operatorname{ch} \xi\left(y-y_{j}\right) \cdot S_{-}\left(y-y_{j}\right)-\frac{\operatorname{sh} \xi\left(l+d_{1}-y_{j}\right)}{\operatorname{sh} \xi\left(2 l+d_{1}+d_{2}\right)} \operatorname{ch} \xi\left(y+l+d_{2}\right)\right)-$

$-\frac{q_{0}}{\xi^{2}} \cdot\left(N(y, l)-\operatorname{ch} \xi(y+l) \cdot S_{-}(y+l)+\operatorname{ch} \xi(y-l) \cdot S_{+}(y-l)\right)+$

$\left.\left.+\frac{\operatorname{sh} \xi\left(2 l+d_{1}\right)-\operatorname{sh} \xi d_{2}}{\operatorname{sh} \xi\left(2 l+d_{1}+d_{2}\right)} \operatorname{ch} \xi\left(y+l+d_{2}\right)\right)\right]+\frac{1}{\xi \operatorname{sh} \xi\left(2 l+d_{1}+d_{2}\right)} \times$

$\times\left[\alpha_{+} \sum_{k=1}^{m-1}\left(t_{k+1}^{(+)}-t_{k}^{(+)}\right) e^{i \xi x_{k}} \operatorname{ch} \xi\left(y+l+d_{2}\right)+\right.$

$\left.\left.+\alpha \sum_{k=1}^{m-1}\left(t_{k+1}^{(-)}-t_{k}^{(-)}\right) e^{i \xi x_{k}} \operatorname{ch} \xi\left(y-l-d_{2}\right)\right]\right\}$. 
Applying the inverse Fourier transform to the relation (12), the following expression for the function $\vartheta$ could be found:

$$
\begin{aligned}
& \vartheta=-\frac{1}{\pi} \cdot\left\{\sum_{j=1}^{n-1}\left(t_{j+1}^{(-l)}-t_{j}^{(-l)}\right) \cdot\left[\lambda_{0}\left(t_{j+1}^{(-l)}\right)-\lambda_{1}\left(t_{j+1}^{(-l)}\right)\right] \int_{0}^{\infty} \frac{\sin \xi\left(x-x_{j}\right)}{\xi} \times\right. \\
& \times\left[(1-\operatorname{ch} \xi(y+l)) S_{-}(y+l)+\frac{\operatorname{sh} \xi\left(2 l+d_{1}\right)}{\operatorname{sh} \xi\left(2 l+d_{1}+d_{2}\right)} \operatorname{ch} \xi\left(y+l+d_{2}\right)\right] d \xi- \\
& -\sum_{j=1}^{n-1}\left[\lambda_{0}\left(t_{j+1}^{(l)}\right)-\lambda_{1}\left(t_{j+1}^{(l)}\right)\right]\left(t_{j+1}^{(l)}-t_{j}^{(l)}\right) \int_{0}^{\infty} \frac{\sin \xi\left(x-x_{j}\right)}{\xi} \times \\
& \times\left[(1-\operatorname{ch} \xi(y-l)) S_{+}(y-l)+\frac{\operatorname{sh} \xi d_{2}}{\operatorname{sh} \xi\left(2 l+d_{1}+d_{2}\right)} \operatorname{ch} \xi\left(y+l+d_{2}\right)\right] d \xi- \\
& -\sum_{j=1}^{n-1}\left(t_{j+1}^{( \pm h)}-t_{j}^{( \pm h)}\right) \cdot\left(\lambda_{0}\left(t_{j+1}^{( \pm h)}\right)-\lambda_{1}\left(t_{j+1}^{( \pm h)}\right)\right) \int_{0}^{\infty} \frac{\sin \xi(x-h)-\sin \xi(x+h)}{\xi} \times \\
& \times\left[\operatorname{ch} \xi\left(y-y_{j}\right) S_{-}\left(y-y_{j}\right)-\frac{\operatorname{sh} \xi\left(l+d_{1}-y_{j}\right)}{\operatorname{sh} \xi\left(2 l+d_{1}+d_{2}\right)} \operatorname{ch} \xi\left(y+l+d_{2}\right)\right] d \xi+ \\
& +q_{0} \int_{0}^{\infty} \frac{\sin \xi(x-h)-\sin \xi(x+h)}{\xi}\left[N(y, l)-\operatorname{ch} \xi(y+l) \cdot S_{-}(y+l)+\right. \\
& \left.+\operatorname{ch} \xi(y-l) \cdot S_{+}(y-l)+\frac{\operatorname{sh} \xi\left(2 l+d_{1}\right)-\operatorname{sh} \xi d_{2}}{\operatorname{sh} \xi\left(2 l+d_{1}+d_{2}\right)} \operatorname{ch} \xi\left(y+l+d_{2}\right)\right] d \xi+ \\
& +\alpha_{+} \sum_{k=1}^{m-1}\left(t_{k+1}^{(+)}-t_{k}^{(+)}\right) \int_{0}^{\infty} \frac{\sin \xi\left(x-x_{k}\right)}{\xi^{2} \cdot \operatorname{sh} \xi\left(2 l+d_{1}+d_{2}\right)} \operatorname{ch} \xi\left(y+l+d_{2}\right) d \xi+ \\
& \left.+\alpha_{-} \sum_{k=1}^{m-1}\left(t_{k+1}^{(-)}-t_{k}^{(-)}\right) \int_{0}^{\infty} \frac{\sin \xi\left(x-x_{k}\right)}{\xi^{2} \cdot \operatorname{sh} \xi\left(2 l+d_{1}+d_{2}\right)} \operatorname{ch} \xi\left(y-l-d_{1}\right) d \xi\right\} \text {. }
\end{aligned}
$$

Substituting the specific subjection coefficients of inclusion materials and strip thermal conduction in the relation (3), (13) and equalizing the expressions of $\vartheta$ function on the edges $K_{ \pm}$and on contact intervals $L_{x}^{ \pm}$, $L_{y}^{ \pm}$the system of nonlinear algebraic equations to determine unknown values of temperature $t_{j}^{( \pm h)}$, $t_{j}^{( \pm l)}(j=\overline{1, n}), t_{k}^{(-)}, t_{k}^{(+)}(k=\overline{1, m})$ is obtained.

The desired temperature field for the nonlinear boundary heat conduction problem (1), (2) is determined from the nonlinear algebraic equation obtained after the application of relations (3), (13) and substitution of the specific expressions for coefficient dependences of thermal conductivity inherent to inclusion and strip materials.

\section{Partial examples and analysis of the results}

There is such a dependency between the coefficient of thermal conductivity and temperature in many practical cases $[6,7]$ :

$\lambda=\lambda^{0} \cdot(1-k t)$, where $\lambda^{0}, k$ are reference and temperature coefficients for the thermal conductivity.

Then, applying expressions (3), (13), the formula to determine the temperature $t$ in areas is obtained $\Omega_{0}=\{(x, y):|x| \leq h,|y| \leq l\}$,

$$
t=\frac{1-\sqrt{1-2 k_{0}\left(\frac{\vartheta}{\lambda_{0}^{0}}+\vartheta_{1}^{*}\right)}}{k_{0}}
$$

$\Omega_{1}=\left\{(x, y):|x|>h,-l-d_{2} \leq y \leq l+d_{1}\right\}$, $\Omega_{2}=\left\{(x, y):|x| \leq h,-l-d_{2} \leq y<-l\right\}$,

$t=\frac{1-\sqrt{1-\frac{2 k_{1} \cdot \vartheta}{\lambda_{1}^{0}}}}{k_{1}}$,

$\Omega_{3}=\left\{(x, y):|x| \leq h, l<y \leq l+d_{1}\right\}$,

$t=\frac{1-\sqrt{1-2 k_{1}\left(\frac{\vartheta}{\lambda_{1}^{0}}+\vartheta_{1}^{*}\right)}}{k_{1}}$,

where

$$
\begin{aligned}
& \vartheta_{1}^{*}=\left.\left\{\left[1-\frac{\lambda_{1}^{0}}{\lambda_{0}^{0}}+\frac{1}{2}\left(\frac{\lambda_{1}^{0}}{\lambda_{0}^{0}} \cdot k_{1}-k_{0}\right) \cdot t\right] \cdot t\right\}\right|_{|x|=h}+ \\
& +\left.\left\{\left[1-\frac{\lambda_{1}^{0}}{\lambda_{0}^{0}}+\frac{1}{2}\left(\frac{\lambda_{1}^{0}}{\lambda_{0}^{0}} \cdot k_{1}-k_{0}\right) \cdot t\right] \cdot t\right\}\right|_{y=-l}+ \\
& +\left.\left\{\left[\frac{\lambda_{1}^{0}}{\lambda_{0}^{0}}-1+\frac{1}{2}\left(k_{0}-\frac{\lambda_{1}^{0}}{\lambda_{0}^{0}} \cdot k_{1}\right) \cdot t\right] \cdot t\right\}\right|_{\substack{|x|=h \\
y=-l}} ;
\end{aligned}
$$$$
\left.t\right|_{|x|=h}=\frac{1-\sqrt{1-\frac{2 k_{1} \cdot \vartheta \mid}{\lambda_{1}^{0}}}}{k_{1}} ;
$$$$
\begin{gathered}
\left.t\right|_{y=-l}=\frac{1-\sqrt{1-\frac{\left.2 k_{1} \cdot \vartheta\right|_{y=-l}}{\lambda_{1}^{0}}}}{k_{1}} ; \\
\left.t\right|_{\substack{|x|=h \\
y= \pm l}}=\frac{1-\sqrt{1-\frac{\left.2 k_{1} \cdot \vartheta\right|_{|x|=h}}{\lambda_{y= \pm l}}}}{k_{1}^{0}} ;
\end{gathered}
$$$$
\left.t\right|_{y=l}=\frac{1-\sqrt{1-2 k_{0}\left(\frac{\left.\vartheta\right|_{y=l}}{\lambda_{0}^{0}}+\vartheta_{3}^{*}\right)}}{k_{0}} ;
$$ 


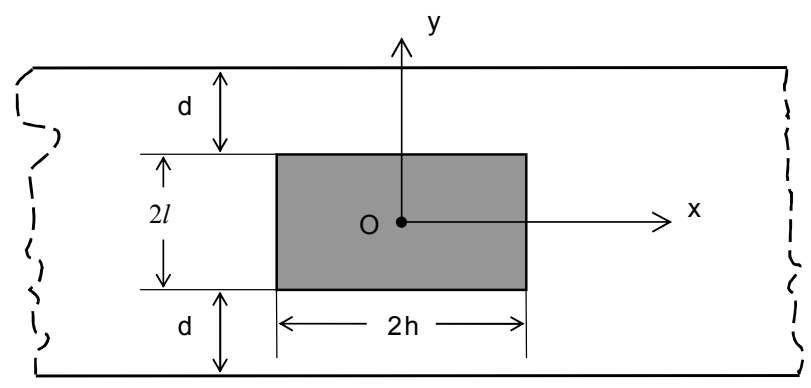

Fig. Thermosensitive strip with rectangular inclusion.

$$
\begin{aligned}
& \vartheta_{2}^{*}=\left.\left\{\left[\frac{\lambda_{0}^{0}}{\lambda_{1}^{0}}-1+\frac{1}{2}\left(k_{1}-k_{0} \cdot \frac{\lambda_{0}^{0}}{\lambda_{1}^{0}}\right) \cdot t\right] \cdot t\right\}\right|_{z=-l}+ \\
& +\left.\left\{\left[1-\frac{\lambda_{0}^{0}}{\lambda_{1}^{0}}+\frac{1}{2}\left(k_{0} \cdot \frac{\lambda_{0}^{0}}{\lambda_{1}^{0}}-k_{1}\right) \cdot t\right] \cdot t\right\}\right|_{z=l}+ \\
& +\left.\left\{\left[\frac{\lambda_{0}^{0}}{\lambda_{1}^{0}}-1+\frac{1}{2}\left(k_{1}-k_{0} \cdot \frac{\lambda_{0}^{0}}{\lambda_{1}^{0}}\right) \cdot t\right] \cdot t\right\}\right|_{\substack{|x|=h \\
z=l}}+ \\
& +\left.\left\{\left[1-\frac{\lambda_{0}^{0}}{\lambda_{1}^{0}}+\frac{1}{2}\left(k_{0} \cdot \frac{\lambda_{0}^{0}}{\lambda_{1}^{0}}-k_{1}\right) \cdot t\right] \cdot t\right\}\right|_{\substack{|x|=h \\
z=-l}} ; \\
& \vartheta_{3}^{*}=\left.\left\{\left[1-\frac{\lambda_{1}^{0}}{\lambda_{0}^{0}}+\frac{1}{2}\left(k_{1} \cdot \frac{\lambda_{1}^{0}}{\lambda_{0}^{0}}-k_{0}\right) \cdot t\right] \cdot t\right\}\right|_{z=-l}+ \\
& +\left.\left\{\left[1-\frac{\lambda_{1}^{0}}{\lambda_{0}^{0}}+\frac{1}{2}\left(k_{1} \cdot \frac{\lambda_{1}^{0}}{\lambda_{0}^{0}}-k_{0}\right) \cdot t\right] \cdot t\right\}\right|_{\substack{|x|=h \\
z=l}}+ \\
& +\left.\left\{\left[\frac{\lambda_{1}^{0}}{\lambda_{0}^{0}}-1+\frac{1}{2}\left(k_{0}-k_{1} \cdot \frac{\lambda_{1}^{0}}{\lambda_{0}^{0}}\right) \cdot t\right] \cdot t\right\}\right|_{\substack{|x|=h \\
z=-l}} .
\end{aligned}
$$

Being based on numerical analysis, it has been determined that it is enough to select the partition number $n$ of the intervals $]-l ; l[]-,h ; 0[$ and $] 0 ; h[$ that equals seven and the partition number $m$ of the interval ] $0 ; x_{*}[$ that equals eleven. Numerical calculations have been performed for the following materials: strip material - ceramics VK-94-1, the inclusion material - tungsten, which show that the consideration of dependency of the thermal conductivity coefficients on temperature leads to reduction of the temperature field compared with non-thermosensitive system (thermaland-physical parameters are independent on temperature) by $3.8 \%$ for selected materials.

\section{Conclusions}

The original nonlinear heat conductivity equation (1) has been partially linearized using the relation (3) that describes a new input function $\vartheta$. The proposed piecewise linear approximation of temperature on the boundaries $L_{x}^{ \pm}, L_{y}^{ \pm}$of an inclusion and at the edges $K_{ \pm}$ of the strip with the expressions (7) that allowed to totally linearize the nonlinear boundary problem of thermal conductivity (5), (6) for a thermosensitive system with the foreign inclusion. An analytical solution to the input function $\vartheta$ has been produced as a formula (3), which enabled to construct new algorithms and developed software to evaluate the temperature gradients in the area of foreign inclusion and to forecast the operation mode of electronic devices with increased thermal resistance and prolonged operating period.

\section{References}

1. Yu.M. Kolyano, Yu.M. Krychevets, E.H. Ivanyk, V.I. Gavrysh, Temperature field in half-space with foreign inclusion // Inzhenerno-fizich. zhurnal (Minsk) 55 (6), p. 1006-1011 (1988) (in Russian).

2. V.I. Gavrysh, V.A. Volos, The problem of the strip with rectangular inclusions heat conductivity // The Lviv Polytechnic State University Bulletin: Applied Mathematics. No. 320, p. 28-33 (1997) (in Ukrainian).

3. Ya.M. Podstryhach, V.A. Lomakin, Yu.M. Kolyano, Thermoelasticity of Heterogeneous Body Structure. Nauka. Moscow, 1984 (in Russian).

4. Yu.M. Kolyano, Methods of Heat Conductivity and Thermoelasticity of Heterogeneous Bodies. Naukova Dumka, Kyiv (1992) (in Russian).

5. D. Fedasyuk, V. Gavrysh, A. Kuzmin, Non-linear heat exchange problem for the piecewise homogeneous strip with foreign inclusion // Proc. Intern. Conference "Modern Problems of Mechanics and Mathematics", vol. 1, Lviv, 2008 (in Ukrainian).

6. V.A. Lomakin, The Theory of Thermoelasticity of Heterogeneous Bodies. Moscow University Publishing House, Moscow, 1976 (in Russian).

7. R. Berman, Heat Conductivity of Solids. Mir, Moscow, 1979 (in Russian). 